\title{
Determination of packaging induced 3D stress utilizing a piezocoefficient mapping device
}

Richter, Jacob; Hyldgård, A.; Birkelund, Karen; Arnoldus, Morten Berg; Hansen, Ole; Thomsen, Erik Vilain

Published in:

20th IEEE International Micro Electro Mechanical Systems (MEMS) Conference

Link to article, DOI:

10.1109/MEMSYS.2007.4433015

Publication date:

2007

Document Version

Publisher's PDF, also known as Version of record

Link back to DTU Orbit

Citation (APA):

Richter, J., Hyldgård, A., Birkelund, K., Arnoldus, M. B., Hansen, O., \& Thomsen, E. V. (2007). Determination of packaging induced $3 \mathrm{D}$ stress utilizing a piezocoefficient mapping device. In 20th IEEE International Micro Electro Mechanical Systems (MEMS) Conference (pp. 69-72). IEEE. https://doi.org/10.1109/MEMSYS.2007.4433015

\section{General rights}

Copyright and moral rights for the publications made accessible in the public portal are retained by the authors and/or other copyright owners and it is a condition of accessing publications that users recognise and abide by the legal requirements associated with these rights.

- Users may download and print one copy of any publication from the public portal for the purpose of private study or research.

- You may not further distribute the material or use it for any profit-making activity or commercial gain

- You may freely distribute the URL identifying the publication in the public portal 


\section{DETERMINATION OF PACKAGING INDUCED 3D STRESS UTILIZING A PIEZOCOEFFICIENT MAPPING DEVICE}

J. Richter, A. Hyldgård, K. Birkelund, M.B. Arnoldus, O. Hansen, and E.V. Thomsen

Technical University of Denmark

Department of Micro and Nanotechnology

Building 345east, DK-2800 Kgs. Lyngby

Telephone: +45 4525 5700. E-mail: jar@mic.dtu.dk

\begin{abstract}
This paper presents a novel method to determine $3 \mathrm{D}$ stress in microsystem packaging. The stress components $\sigma_{\mathrm{xx}}, \sigma_{\mathrm{yy}}, \sigma_{\mathrm{zz}}$, and $\sigma_{\mathrm{xy}}$ are found in an epoxy package using a piezocoefficient mapping device as stress sensor. We spin the current $360^{\circ}$ in a circular $n$-type (001) Si piezoresistor by contacts located near the perimeter of the resistor and do high impedance voltage measurements on contacts located near the centre of the resistor. By measuring the potential drops in these contacts we can determine the stress in the chip. The epoxy is potted in a polystyrene tube using the same concept as in [1] used for chip packaging for fisheries research. We investigate the EpoTek 305 epoxy and find stress values of $\sigma_{\mathrm{xx}} \approx-23 \mathrm{MPa}, \sigma_{\mathrm{yy}} \approx-1 \mathrm{MPa}$, $\sigma_{\mathrm{xy}} \approx 0.3 \mathrm{MPa}$, and $\sigma_{\mathrm{zz}} \approx 40 \mathrm{MPa}$. The presented method can be used for $3 \mathrm{D}$ stress measurements of various packaging concepts.
\end{abstract}

\section{INTRODUCTION}

The last step to commercialisation of a MEMS device is often related to packaging. Packaging protects the device from the surroundings. A difficult task is to protect the device and not affect the performance of the device at the same time. The performance of an electrical component can be highly influenced by packaging induced stress. Stress sensors are used to measure the stress and thus explain the performance of the device. In ref. [2] a CMOS integrated stress sensor for packaged integrated circuit dies is presented, and ref. [3] shows a van der Pauw structure used for stress sensing.

In this paper we present a method to measure four components of the stress, $\sigma_{\mathrm{xx}}, \sigma_{\mathrm{yy}}, \sigma_{\mathrm{zz}}$, and $\sigma_{\mathrm{xy}}$. This method makes complex stress analysis possible in microscale packaging. The packaging concept analysed is used to protect chip and electronics in a multisensor system developed for fisheries research [4].

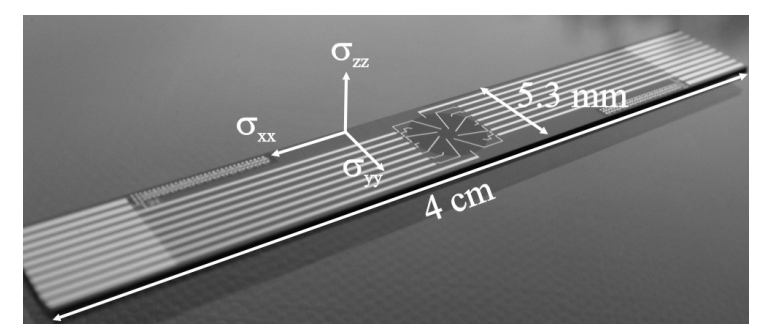

Figure 1 Presentation of stress sensor. The chip is cut along the direction directed $22.5^{\circ}$ with respect to the [100] direction. In the centre region of the chip a single circular $n$-type resistor is implemented. A current is forced through the resistor by contacts near the perimeter and 8 contacts placed in a radius of $100 \mu \mathrm{m}$ with respect to the resistor centre measure 4 potential drops.

\section{THEORY}

Inspired by the work of Bartholomeyczik [5] and Steiner [6] we have designed a (001) n-type $\mathrm{Si}$ circular piezoresistor. The piezoresistor is placed in the centre region of a long thin chip. The chip is dimensioned to fit in a four point bending (4PB) fixture [7]. Figure 1 shows a photograph of the chip. The chip is cut along the direction of $22.5^{\circ}$ with respect to the [100] direction. A conceptual drawing of the device is shown in Figure 2. We direct the current, $I(\phi)$, at an angle $\phi$ by using four outer contacts placed near the resistor perimeter. Thus, in the centre of the circular piezoresistor we spin the current $360^{\circ}$.

In the centre of the resistor at a radius of $100 \mu \mathrm{m}$ eight contacts are placed. The potential drops, $V_{\mathrm{i}}=$ $V_{\text {ia }}-V_{\mathrm{ib}}$, are measured across the inner contacts where $i=1,2,3,4$, see Figure 2. Thus, for each current direction four potential drops are measured. To a first order approximation the relative change, $\Delta V_{\mathrm{i}} / V_{\mathrm{i}, 0}$, where $V_{\mathrm{i}, 0}$ is the potential drop $V_{\mathrm{i}}$ at zero stress, is equal to a linear combination of the stress components. Defining two fictive voltage drops, $V_{\mathrm{p}}$ and $V_{\mathrm{t}}$ in Figure 2, as linear combinations of the four potential drops we obtain a condensed linear system where the number of current directions determines the number of equations

$$
\begin{aligned}
V_{p}= & \frac{1}{2}\left(\cos (\phi) \cdot V_{1}+\cos \left(\phi-\frac{\pi}{4}\right) \cdot V_{2}\right. \\
& \left.+\sin (\phi) \cdot V_{3}+\sin \left(\phi-\frac{\pi}{4}\right) \cdot V_{4}\right) \\
V_{t}= & \frac{1}{2}\left(\sin (\phi) \cdot V_{1}+\sin \left(\phi-\frac{\pi}{4}\right) \cdot V_{2}\right. \\
& \left.-\cos (\phi) \cdot V_{3}-\cos \left(\phi-\frac{\pi}{4}\right) \cdot V_{4}\right)
\end{aligned}
$$


Table 1 Relations of selected relative potential drops. For $\Delta V_{2} / V_{2,0}$ and $\Delta V_{4} / V_{4,0}$ similar equations are valid.

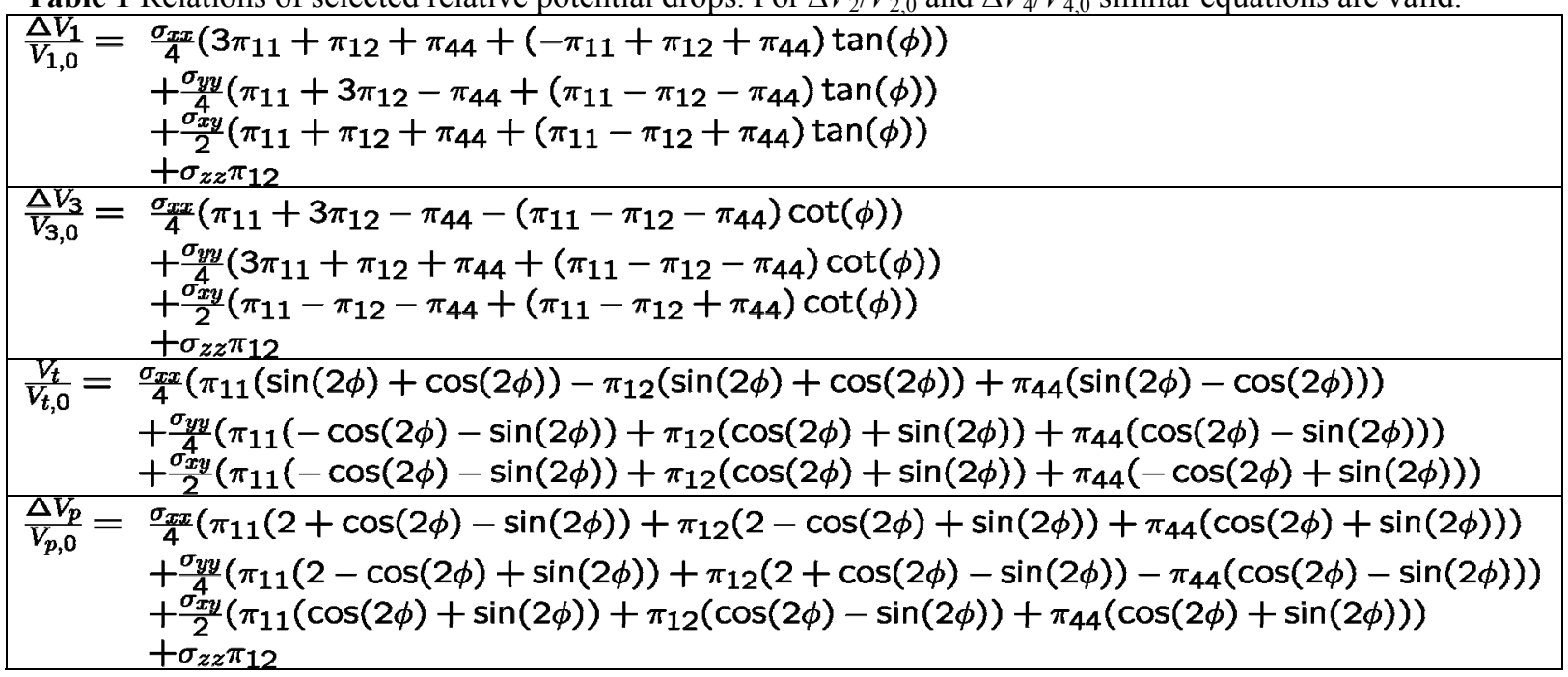

where $\phi$ is the angle between the current direction and the chip-axis. Table 1 shows the expected relations of $\Delta V_{1} / V_{1,0}, \quad \Delta V_{3} / V_{3,0}, \quad \Delta V_{\mathrm{p}} / V_{\mathrm{p}, 0}, \quad$ and $\Delta V_{\mathrm{t}} / V_{\mathrm{p}, 0}$. Similar equations are valid for $\Delta V_{2} / V_{2,0}$, and $\Delta V_{4} / V_{4,0}$.

As seen in Table 1 the relative voltage changes depend on the angle $\phi$, the stress components $\sigma_{\mathrm{xx}}$, $\sigma_{\mathrm{yy}}, \sigma_{\mathrm{xy}}$, and $\sigma_{\mathrm{zz}}$, and the piezocoefficients $\pi_{11}, \pi_{12}$, and $\pi_{44}$. Performing the measurements when the chip is located in the 4PB fixture we can determine the three piezocoefficients. This can be done since the 4PB fixture applies a well-defined uniform and uniaxial stress, $\sigma_{\mathrm{xx}} \neq 0$, to the centre region of the chip where the resistor is placed [7].

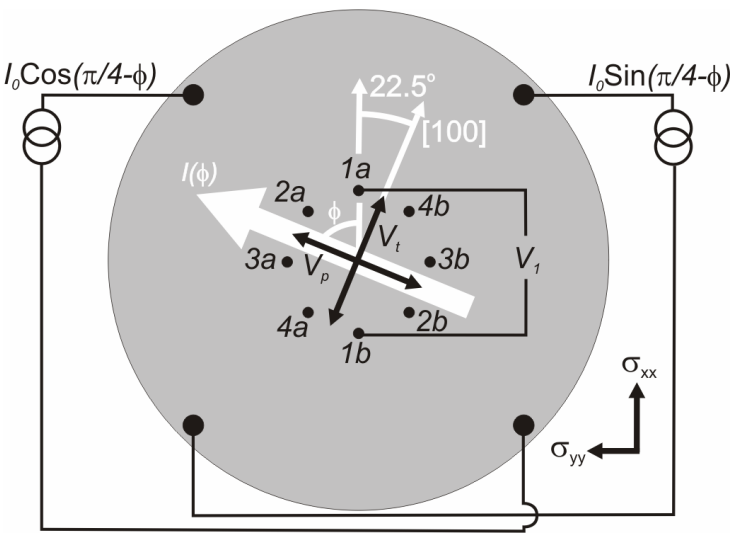

Figure 2 Schematic of the circular piezoresistor. The illustration is not to scale. The resistor diameter is $1700 \mu \mathrm{m}$ and the inner contacts are placed in a circle with a radius of $100 \mu \mathrm{m}$. The four outer contacts spin the current $360^{\circ}$ in the centre of the resistor. The fictive voltage drops, $V_{\mathrm{p}}$ and $V_{\mathrm{t}}$, are linear combinations of all measured potential drops, $V_{\mathrm{i}}=V_{\mathrm{ia}}-V_{\mathrm{ib}}$ where $i=1,2,3,4$. The potential drop $V_{1}$ is showed as an example.
After this calibration step the chip is packaged and the measurements are performed again. Setting up the linear equation system of $V_{\mathrm{p}}$ and $V_{\mathrm{t}}$ we can determine the four stress components

$$
\frac{\Delta V}{V_{0}}=\sigma_{x x} \cdot a(\phi)+\sigma_{y y} \cdot b(\phi)+\sigma_{x y} \cdot c(\phi)+\sigma_{z z} \cdot d(\phi)
$$

where $a, b, c$, and $d$ are functions of $\phi$ and contains linear combinations of $\pi_{11}, \pi_{12}$, and $\pi_{44}$. The number of equations is determined by the number of spinning angles, $\phi$.

\section{EXPERIMENTAL}

The stress sensor chip is fabricated in a cleanroom environment using microtechnology. A crosssection of the device is shown in Figure 3. The resistors contain a doping concentration of $N_{\mathrm{D}}=10^{18} \mathrm{~cm}^{-3}$ implemented by phosphorous ion implantation on a $p$-type (001) silicon substrate. The resistors are defined using a reactive ion etch and a thermal oxidation activates the donors and ensures isolation between resistor and metal. The metallization occurs in a lift-off process with a multi layer of $\mathrm{Ti}$ and Al. The final fabrication step separates the chips using advanced silicon etch. This allows for off-axis dicing.

The electrical measurements are performed with a four point measurement. In Figure 3 it is illustrated how the current is forced through the contacts near the resistor perimeter while performing a high impedance voltage measurement on the contacts localized close to the centre of the resistor. The inner contacts are placed in a radius of $100 \mu \mathrm{m}$ with respect to the resistor centre ensuring a uniform current distribution in the area of the contacts. 


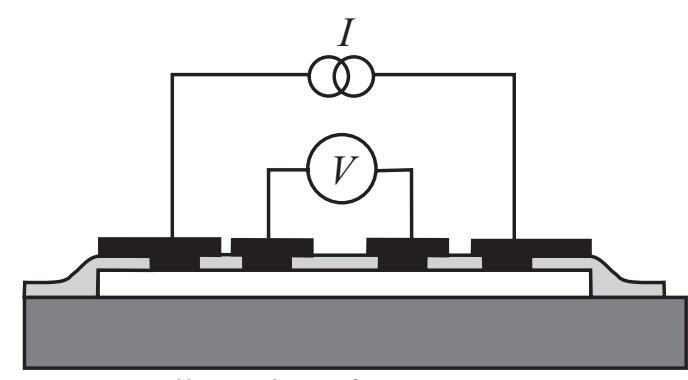

Figure 3 Illustration of stress sensor cross section and measurement principle. The $n$-type resistor (white color) is fabricated by phosphorous implantation on a $p$-type substrate (dark grey). The metal contacts (black) consist of a Ti/Al multi layer and a thermal oxide (light grey) is used for isolation. The current is forced through the resistor from contacts near the resistor perimeter and a high impedance voltage measurement is performed on the contacts near the resistor centre.

\section{Calibration}

The original use of the chip is to map the piezocoefficients by spinning the current on a resistor exposed to a defined uniaxial and uniform stress [7]. This is done in a state-of-the-art 4PB fixture shown in Figure 4. Figure 5 shows the relative change in voltage $V_{1}$ divided by the applied stress, $\sigma_{x x}$, as a function of $\tan (\phi)$. From Table 1 we find that the slope and the offset of the linear fit are related to the three piezocoefficients.

We determine $\pi_{11}, \pi_{12}$, and $\pi_{44}$ from the overdetermined linear equation system obtained by the relations for the other potential drops in Table 1 .

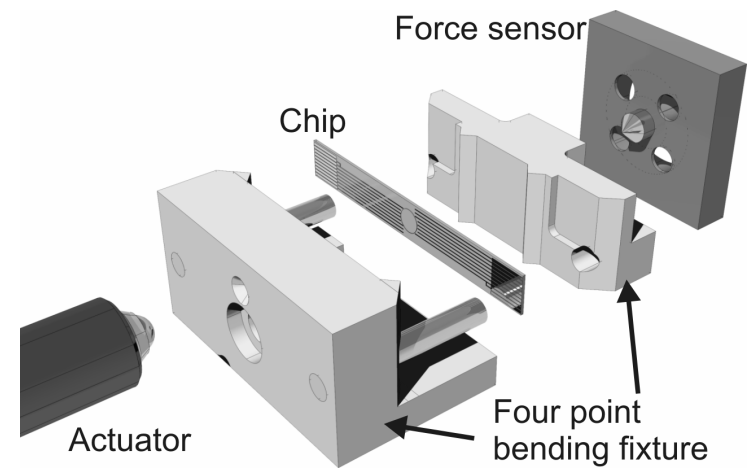

Figure 4 Illustration of the state-of-the-art four point bending fixture used for calibration of the piezocoefficient mapping device. The chip is placed in between the two sets of blades and an actuator is pushing the two parts together. The force on the chip is measured by a highly sensitive force sensor. The fixture applies a uniaxial and uniform stress in the centre region of the chip.

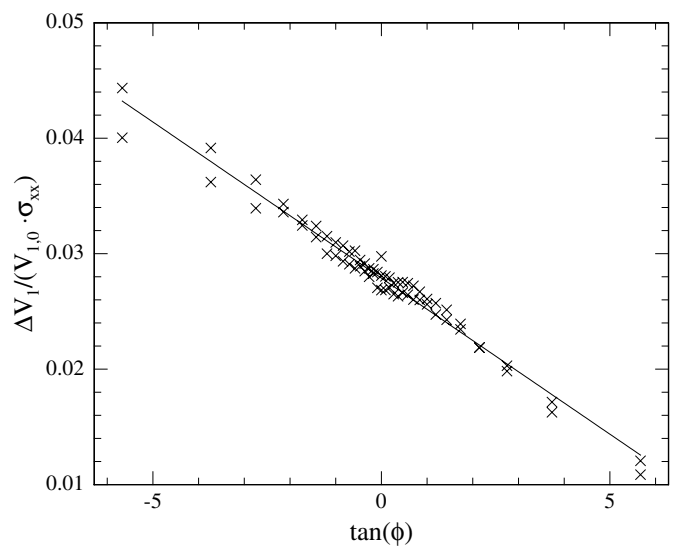

Figure 5 Data of the relative voltage drop measured on $V_{1}, \Delta V_{1} / V_{1,0}$, divided by the applied stress, $\sigma_{\mathrm{xx}}$. Using the relation in Table 1 and the fitted values of the slope and the offset we can determine the three piezocoefficients by including a second equation, e.g. the relation for $\Delta V_{\mathrm{p}} / V_{\mathrm{p}, 0}$.

\section{Packaging}

After the piezocoefficients have been determined, the chip is potted with EpoTek 305 two-component epoxy in a polystyrene tube. Figure 6 is a photograph of the packaged chip after curing. We perform the same electrical measurements on the packaged chip as performed in the four point bending fixture. Figure 7 shows the relative voltage change $\Delta V_{3} / V_{3,0}$ as a function of $\cot (\phi)$.

The fictive voltages $V_{\mathrm{p}}$ and $V_{\mathrm{t}}$ are calculated as shown in Equation (1). The linear equation system in Equation (2) can then be written for both voltages with the relations in Table 1 and the stress components are extracted.

\section{RESULTS}

The stress has been measured in three samples. The results are presented in Table 2. The largest stress component is the stress normal to the surface plane, $\sigma_{\mathrm{zz}} \approx 40 \mathrm{MPa}$ with an ucertainty of $\Delta \sigma_{\mathrm{zz}} \approx 1.5 \mathrm{MPa}$. The stress component along the chip edge is $\sigma_{\mathrm{xx}} \approx-23 \mathrm{MPa}$. The two other stress components $\sigma_{\mathrm{yy}} \approx 1 \mathrm{MPa}$ and $\sigma_{\mathrm{xy}} \approx-0.3 \mathrm{MPa}$ are small compared to these values. Although the uncertainty is comparable to the measured stress values we conclude that $\sigma_{\mathrm{yy}}$ and $\sigma_{\mathrm{xy}}$ are small compared to $\sigma_{\mathrm{xx}}$ and $\sigma_{\mathrm{zz}}$. The fact that $\sigma_{\mathrm{xx}}$ is much larger than $\sigma_{\mathrm{yy}}$ can be explained by the asymmetry of the polystyrene tube and the shape of the chip. 


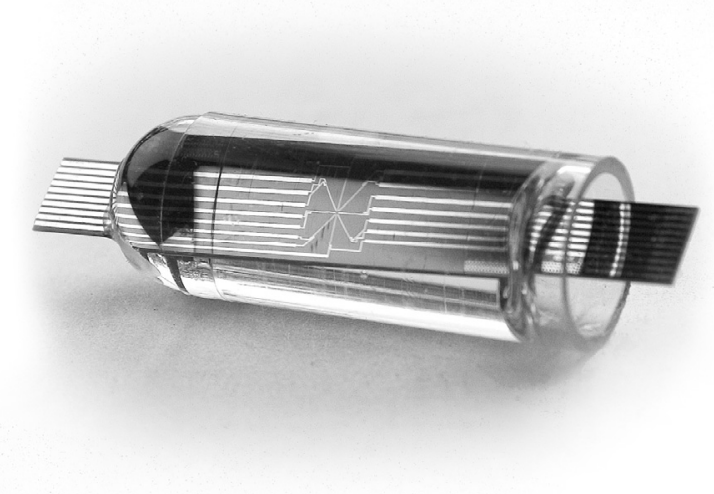

Figure 6 Photograph of the packaged device. The circular piezoresistor is placed in the centre region of the chip. A two-component epoxy is potted in a polystyrene tube. The packaging concept is used in ref. [1] for fisheries research and allows direct exposure of one end of a chip while sealing the other end (along with electronics, battery, etc.) from the surroundings.

The $\sigma_{x x}$ component depends on the placement of the chip in the tube. This explains the relative large variation in the measured values.

In the hardening process the epoxy is contracted. This contraction contributes to the value of $\sigma_{\mathrm{zz}}$. The variation of the measured value of this component is small since the same epoxy was used for all samples.

\section{CONCLUSION}

We have presented a method which enables 3D stress characterization. To show the concept the stress sensors have been tested in a polystyrene tube potted with the two-component epoxy, EpoTek 305. The results show large stress components along and normal to the chip, $\sigma_{\mathrm{xx}} \approx-23 \mathrm{MPa}$ and $\sigma_{\mathrm{zz}} \approx 40 \mathrm{MPa}$. The two other components, $\sigma_{\mathrm{yy}} \approx 1 \mathrm{MPa}$ and $\sigma_{\mathrm{xy}} \approx-0.3 \mathrm{MPa}$, are small compared to these values and lies within the uncertainty interval.
The method has proven to extract the four stress components, $\sigma_{\mathrm{xx}}, \sigma_{\mathrm{yy}}, \sigma_{\mathrm{zz}}$, and $\sigma_{\mathrm{xy}}$. For future stress analysis we are thus able to perform detailed 3D measurements of packaging induced stress in microsystems.

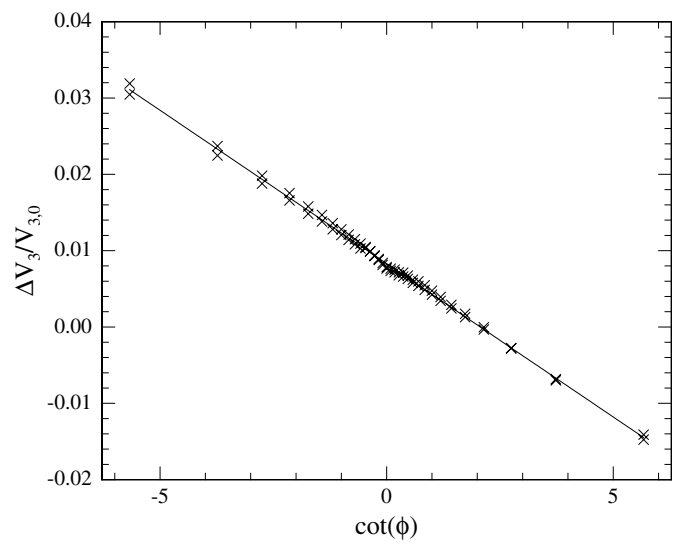

Figure 7 The relative voltage change of $V_{3}$ as a function of $\cot (\phi)$. The slope and the offset of the linear fit are related to the stress components as listed in Table 1.

\section{REFERENCES}

[1] A. Hyldgård, et al, Proc. Eurosensors 2006, pp. 262-263.

[2] P. Ruther, et al, Proc. IEEE Sensors 2006, pp. 314.

[3] A. Mian, J.C. Suhling, R.C. Jaeger, IEEE Sensors Journal, 6-2 (2006), pp. 340-356.

[4] A. Hyldgård, O. Hansen and E. V. Thomsen, Proc. IEEE MEMS 2005, pp. 303-306.

[5] J. Bartholomeyczik, et al, Proc. IEEE MEMS 2004, pp. 263-266.

[6] R. Steiner, et al, Sensors and Actuators A, 66 (1998), pp. 167-172.

[7] M.B. Arnoldus, et al, Proc. Eurosensors 2006, pp. 378-379.

Table 2 Measured values of the stress components, $\sigma_{\mathrm{xx}}, \sigma_{\mathrm{yy}}, \sigma_{\mathrm{zz}}$, and $\sigma_{\mathrm{xy}}$ induced by epoxy and the estimated uncertainties.

\begin{tabular}{|c||c|c||c|c||c|c||c|c|}
\hline Type & $\begin{array}{c}\sigma_{\mathrm{xx}} \\
{[\mathrm{MPa}]}\end{array}$ & $\begin{array}{c}\Delta \sigma_{\mathrm{xx}} \\
{[\mathrm{MPa}]}\end{array}$ & $\begin{array}{c}\sigma_{\mathrm{yy}} \\
{[\mathrm{MPa}]}\end{array}$ & $\begin{array}{c}\Delta \sigma_{\mathrm{yy}} \\
{[\mathrm{MPa}]}\end{array}$ & $\begin{array}{c}\sigma_{\mathrm{zz}} \\
{[\mathrm{MPa}]}\end{array}$ & $\begin{array}{c}\Delta \sigma_{\mathrm{zz}} \\
{[\mathrm{MPa}]}\end{array}$ & $\begin{array}{c}\sigma_{\mathrm{xy}} \\
{[\mathrm{MPa}]}\end{array}$ & $\begin{array}{c}\Delta \sigma_{\mathrm{xy}} \\
{[\mathrm{MPa}]}\end{array}$ \\
\hline \hline EpoTek 305 & -26.9 & 1.2 & -0.7 & 1.2 & 39.7 & 1.4 & -0.4 & 0.2 \\
\hline EpoTek 305 & -20.1 & 0.8 & -0.4 & 0.7 & 40.5 & 0.9 & -0.2 & 0.2 \\
\hline EpoTek 305 & -23.1 & 3.2 & 2.4 & 2.8 & 38.6 & 3.5 & 0.5 & 0.6 \\
\hline
\end{tabular}

